\title{
Parallel Susceptibility of Thin Ferromagnetic Films within Many-Body Green's Function Theory
}

\author{
V. ILKOVIČ \\ Department of Theoretical Physics and Astrophysics, Faculty of Science \\ P.J. Šafarik University, Jesenná 5, 04001 Košice, Slovak Republic \\ AND J. KECER \\ Department of Physics, Technical University \\ Park Komenského 2, 04001 Košice, Slovak Republic

\begin{abstract}
Parallel susceptibility of the quantum Blume-Capel model for ferromagnetic films is calculated within the Green function theory. The Hamiltonian includes a Heisenberg term with the different surface exchange coupling with respect to bulk, an external magnetic field, a second-order uniaxial single-ion anisotropy, and the exchange anisotropy. The importance of collective excitations is demonstrated by comparing with result calculated within mean field approximation.
\end{abstract}

PACS numbers: 75.10.Jm, 75.30.Ds, 75.30.Kz, 75.70.Ak

\section{Model and method}

Among the different experimental methods the measurement of the susceptibility $\chi(T)$ is a very powerful method for the analysis of thin film systems [1, 2]. The maximum of the susceptibility corresponds to the occurrence of a nonvanishing magnetization for the temperatures below the Curie temperature $T_{\mathrm{C}}$. It is known from general considerations [3] that the mentioned maximum of $\chi(T)$ is only observed if the susceptibility is measured along the easy axis, which we denote by "parallel" susceptibility. With the help of a Heisenberg model solved within the Green function theory (GFT) it is possible to perform a quantitative comparison with experiment. Jensen et al. [4] measured the influence of the magnetic anisotropy on the parallel susceptibility of ultrathin Co films grown on $\mathrm{Cu}$ substrate. By comparison with a theoretical analysis of the parallel susceptibility 
in the framework of GFT they determined the parameters of the isotropic exchange interaction and magnetic anisotropy. Using achieved parameters quantitative agreement between theory and experiment was obtained. In recently appeared work [5] there have been studied phase diagrams of the quantum Blume-Capel model. In the present paper we modify this work on the calculation within GFT and mean field approximation (MFA) the parallel susceptibility $\chi_{z z}$ with respect to the easy $z$-axis.

We consider the ferromagnetic films with the Hamiltonian consisting of an isotropic Heisenberg exchange interaction with strength $J_{i j}>0$ between nearest neighbour lattice sites, an exchange anisotropy with strength $D>0$, a secondorder single-ion anisotropy with strength $K_{2}>0$ and the magnetic field with strength $h^{z}$ in convention units

$$
\begin{aligned}
\mathrm{H}= & -\frac{1}{2} \sum_{\langle i, j\rangle} J_{i j}\left(S_{i}^{-} S_{j}^{+}+S_{i}^{z} S_{j}^{z}\right) \\
& -\frac{1}{2} D \sum_{\langle i, j\rangle} S_{i}^{z} S_{j}^{z}+K_{2} \sum_{i}\left(S_{i}^{z}\right)^{2}-h^{z} \sum_{i} S_{i}^{z} .
\end{aligned}
$$

Here the notation $S_{k}^{ \pm}=S_{k}^{x} \pm \mathrm{i} S_{k}^{y}(k=i, j)$ is introduced, where $i$ and $j$ are lattice sites indices and $\langle i j\rangle$ indicates summation over nearest neighbours only, $K_{2}$ takes the different values: $K_{2}(1)$ on the odd layers and $K_{2}(2)$ on the even ones and the exchange parameter takes the value $J_{S}$ at the surfaces and $J$ inside of the film.

In order to treat the problem for general spin $S$, we need the following Green functions $(\mathrm{GFs}) G_{i j}^{l}(\omega)=\left\langle\left\langle S_{i}^{+} ;\left(S_{j}^{z}\right)^{l} S_{j}^{-}\right\rangle\right\rangle_{\omega}$, where $l \geq 0$ is integer, necessary for dealing with higher spin values $S$. These GFs are solved in the usual way by the equation of motion [6]. The higher-order GFs appearing within this procedure are approximated by the Tyablikov-decoupling. For the terms stemming from the single-ion anisotropy we have chosen the Anderson-Callen decoupling procedure [7]. Using the eigenvector method (EVM) described, for example in [8], after Fourier transform to two-dimensional momentum space $\boldsymbol{q}$ (a wave vector in the planes parallel to the film surface), $L$ coupled equations of motion for GFs $G_{\nu \mu}^{(l)}(\boldsymbol{q}, \omega)$ of layer labeled by $\mu$ can be written in the matrix form as

$$
\left(\omega \mathbf{1}-\boldsymbol{P}_{L}\right) \boldsymbol{G}_{\mu}^{(l)}=\boldsymbol{A}_{\mu}^{(l)}, \quad \mu=1, \ldots L,
$$

where $\mathbf{1}$ is the $L \times L$ unit matrix, $\boldsymbol{P}_{L}$ is the matrix of the set of $L$ equations of motion, $\boldsymbol{G}_{\mu}^{(l)}(\boldsymbol{q}, \omega)$ is the Green function vector with components $G_{1 \mu}^{(l)}(\boldsymbol{q}, \omega), \cdots, G_{L \mu}^{(l)}(\boldsymbol{q}, \omega)$ where $\boldsymbol{A}_{\mu}^{(l)}$ is the inhomogeneity vector with components $A_{\mu \mu}^{(l)}=\left\langle\left\langle\left[S_{\mu \mu}^{+},\left(S_{\mu \mu}^{z}\right)^{l} S_{\mu \mu}^{-}\right]\right\rangle\right\rangle$. By using the spectral theorem, for the spontaneous magnetization per site in each atomic layer of the film with spin $S$ we obtain

$$
\left\langle S_{\mu}^{z}\right\rangle_{S}=\frac{\left(S-\Phi_{\mu \mu}\right)\left(1+\Phi_{\mu \mu}\right)^{2 S+1}+\left(1+S+\Phi_{\mu \mu}\right) \Phi_{F \mu \mu}^{2 S+1}}{\left(1+\Phi_{\mu \mu}\right)^{2 S+1}-\Phi_{\mu \mu}^{2 S+1}},
$$

where $\Phi_{\mu \mu}=\frac{1}{\pi^{2}} \int_{0}^{\pi} \int_{0}^{\pi} \mathrm{d} q_{x} \mathrm{~d}_{y} \sum_{\nu=1}^{L} \sum_{\kappa=1}^{L} R_{\mu \nu} \mathcal{E}_{\nu \kappa} \delta_{\nu \kappa} L_{\kappa \mu} . \quad \boldsymbol{R}$ is matrix whose 
columns are the right eigenvectors of matrix $\boldsymbol{P}_{L}$, its inverse $\boldsymbol{L}=\boldsymbol{R}^{-1}$ contains the left eigenvectors as rows, $\mathcal{E}_{\nu \kappa} \delta_{\nu \kappa}=\left(\mathrm{e}^{\beta \omega_{\nu}}-1\right)^{-1}$ are matrix elements of a diagonal matrix $L \times L, \beta=1 / k_{\mathrm{B}} T$ and $\omega_{\nu}$ are the eigevalues of matrix $\boldsymbol{P}_{L}$. From (3) the susceptibilty $\chi_{z z}$ along the easy axis, will be determined numerically as differential quotients $\chi_{z z}=\frac{\left\langle S^{z}\left(h^{z}\right)\right\rangle-\left\langle S^{z}(0)\right\rangle}{h^{z}}$.

\section{Results}

Now we would like to demonstrate the behavior of the inverse easy axis susceptibility $\chi_{z z}^{-1}$ in a large temperature range above reduced Curie temperature $k_{\mathrm{B}} T_{\mathrm{C}}^{f} / J$ of the thin film within GFT and MFA. In Fig. $1 \mathrm{~A}$ there is plotted $\chi_{z z}^{-1}$ as a

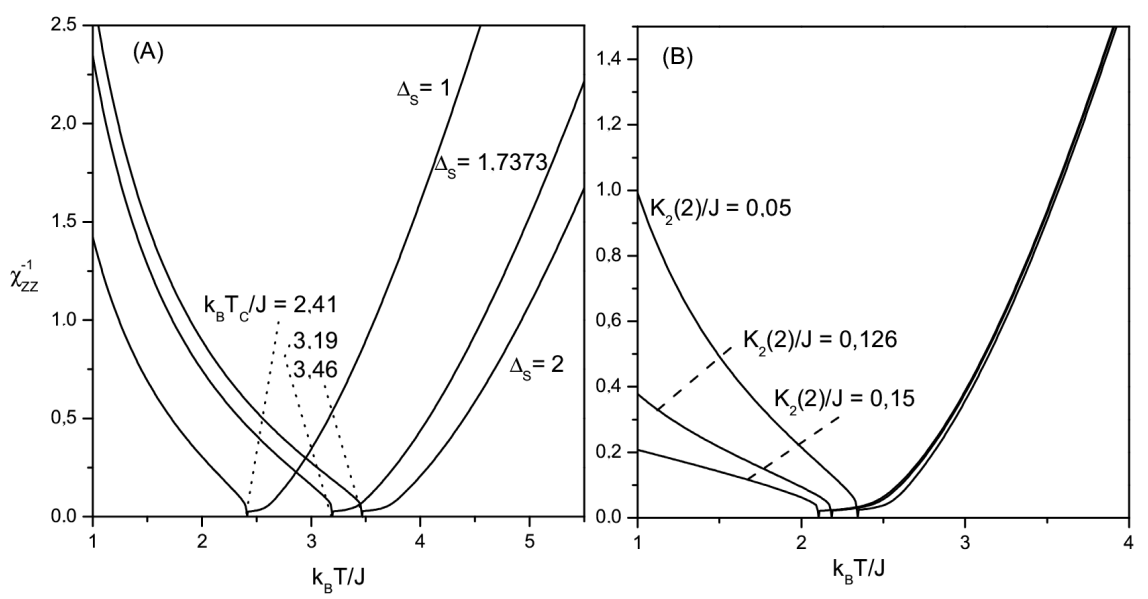

Fig. 1. Function $\chi_{z z}^{-1}(T)$ calculated within GFT for the thin film with the parameters listed in the text: (A) for different values of $\Delta_{S}$, and (B) for different values of the parameter $K_{2}(2) / J$.

function of the reduction temperature $k_{\mathrm{B}} T / J$ calculated within GFT for thin film with spin $S=1$ and with thickness $L=3$ for different values of $\Delta_{S}=J_{S} / J$ when the reduced magnetic strength $h^{z} / J=0.01$, the reduced exchange anisotropy in $z$-direction $D / J=0.01$ and the reduced anisotropic parameters $K_{2}(1) / J=0.005$, $K_{2}(2) / J=0.01$. In Fig. $1 \mathrm{~B}$ the function $\chi_{z z}^{-1}(T)$ is shown for different values of the anisotropy parameter $K_{2}(2) / J$ when $\Delta_{S}=1$ and $K_{2}(1) / J=0.005 . \chi_{z z}^{-1}(T)$ vanishes at $T=T_{\mathrm{C}}^{f}$. The function $\chi_{z z}^{-1}(T)$ in both cases calculated within GFT are curved owing to the presence of spin waves, whereas the corresponding MFA calculation shows linear in the temperature behavior for $T>T_{\mathrm{C}}$ (see Fig. 2). 

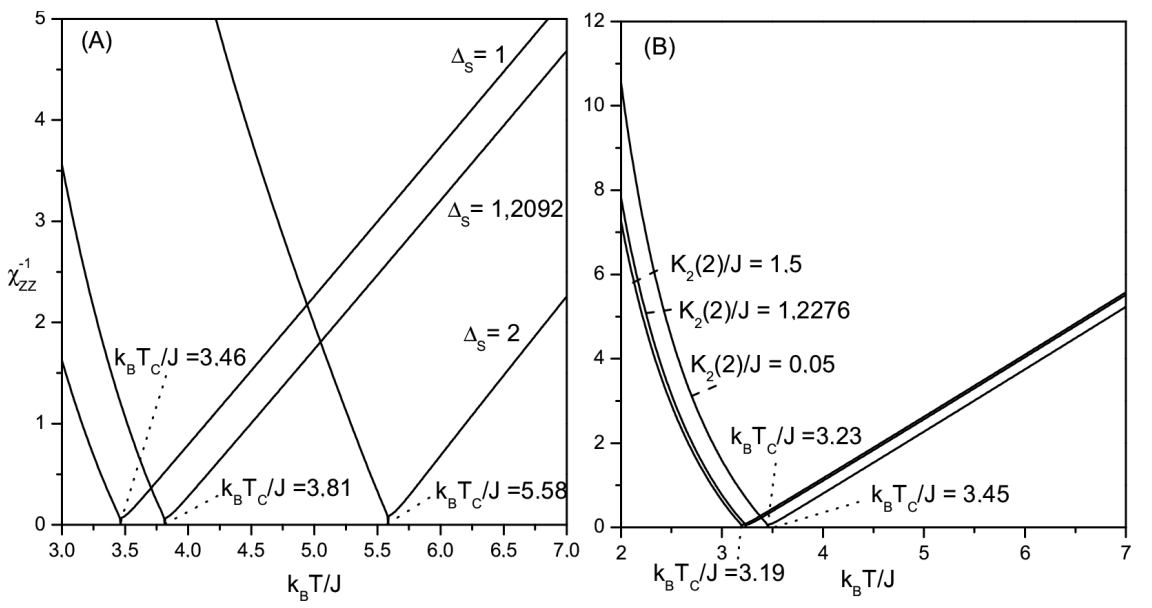

Fig. 2. Function $\chi_{z z}^{-1}(T)$ calculated within MFA for the thin film with the same parameters as in Fig. 1 for different values of $\Delta_{S}$ (Fig. 2A) and as in Fig. 1B for different values of $K_{2}(2) / J$ (Fig. $\left.2 \mathrm{~B}\right)$.

\section{Acknowledgments}

Part of this work (V.I.) was carried out at the JINR, Dubna. Financial support of this work was provided by grant 1/2008/05, Grant Agency for Science, Slovak Republic.

\section{References}

[1] D. Venus, C.S. Arnold, M. Dunlavy, Phys. Rev. B 60, 9607 (1999).

[2] F. Beusch, G. Gerreau, R. Moosbühler, G. Bayreuther, J. Appl. Phys. 89, 7133 (2001).

[3] P. Pfeuty, G. Toulouse, Introduction to the Renormalization Group and to Critical Phenomena, Wiley, London 1977.

[4] P.J. Jensen, S. Knappmann, W. Wulfhekel, H.P. Oepen, Phys. Rev. B 67, 184417 (2003).

[5] J. Kecer, S. Tuleja, Acta Phys. Pol. A 113, 197 (2007).

[6] W. Gasser, E. Heiner, K. Elk, Greensche Funktionen in Festkörper- und Vielteilchenphysik, Wiley-VCH Verlag, Berlin 2001, p. 21.

[7] H.B. Callen, Phys. Rev. 130, 890 (1963).

[8] P. Fröbrich, P.J. Kuntz, Phys. Rep. 432, 223 (2006). 